\title{
Nonlinear Control of Autonomous Flying Cars with Wings and Distributed Electric Propulsion
}

\author{
Xichen Shi, Kyunam Kim, Salar Rahili, and Soon-Jo Chung
}

\begin{abstract}
Hybrid vertical take-off and landing vehicles (VTOL) with lift production from wings and distributed propulsive system present unique control challenges. Existing methods tend to stitch and switch different controllers specially designed for fixed-wing aircraft or multicopters. In this paper, we present a unified framework for designing controllers for such winged VTOL vehicles that are commonly found in recent flying car models. The proposed method is broken down into nonlinear control of both position and attitude with forces and moments as inputs, and real-time control allocation that integrates distributed propulsive actuation with conventional control surface deflection. We also present a strategy that avoids saturation of distributed propulsion control inputs. The effectiveness of the proposed framework is demonstrated through simulation and closed-loop flight experiment with our winged VTOL flying car prototype.
\end{abstract}

\section{INTRODUCTION}

The idea of vertical take-off and landing (VTOL) vehicles has been conceived by inventors well before human made our maiden powered flight. The most recent surge began when miniaturization of power, propulsion, computation, and sensor technologies popularized multicopter. The canonical form of multicopter uses an even number of symmetric, coplanar rotors to generate thrust. Despite being mechanically simple to build and control, they suffer from the fundamental limitations of a rotary wing system: its power inefficiency for forward flight. Throughout history, there have been designs of VTOL-capable aircraft that include wing surfaces to assist in lift production. Some human-piloted commercial designs incorporate engines with vectored thrust output onto fixedwing aircraft. Recent interest in urban aerial transportation calls for a new vehicle design that can take-off and land in a cluttered urban environment while efficiently flying a good range over a metropolitan area [1]. Now often referred to as "flying cars" by the public, the concept of aerial urban transporter naturally requires both efficiency of fixed wings and maneuverability of VTOLs. Some of the popular types include: (1) copter-plane: a direct hybrid between planes and multicopters; (2) tilt-rotor/vectored thrust aircraft: thrust can be diverted in different directions; and (3) tail-sitter: thruster is situated in the back, and the aircraft takes off upright and transition to horizontal flight.

Position trajectory tracking for aerial vehicles rely on the vehicles' ability to generate forces in desired directions. Although the control of aerial vehicles often rely on a property of timescale separation in the flight dynamics [2], [3],

Xichen Shi, Kyunam Kim, Salar Rahili, and Soon-Jo Chung are with the Department of Aerospace (GALCIT) at California Institute of Technology, Pasadena, CA, USA. Email: xshi@caltech.edu, knkim@caltech.edu, srahili@caltech.edu, sjchung@caltech.edu.

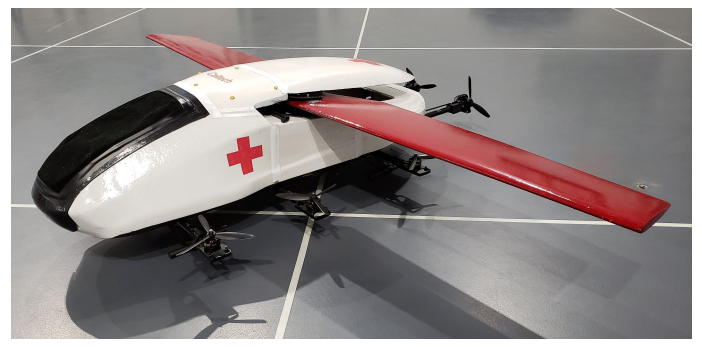

Fig. 1. Caltech's VTOL flying ambulance (1/5 scale) that includes distributed electric propulsion, tilt-able rear rotors and a main wing.

the key difference results from what forces are considered significant during control design. In both fixed-wing and VTOL multicopters, the collective force from all thrusters is one-dimensional. In other words, a multicopter points in the direction of the total commanded force whereas a fixed-wing aircraft merely uses thrust to cancel drag force and relies on a lift force for maneuver. In the case of a winged VTOL, the controller should realize these potentials as much as possible to extend the flight envelope. The problem becomes more challenging when the vehicle can produce thrust in multiple directions by using Distributed Electric Propulsion (DEP) systems [4], [5].

Related Work. Most related work focuses on particular types of VTOL aircraft. Tilt-rotor controllers were designed around the pre-determined rotor tilt angles using model predictive control or adaptive control methods [6], [7]. Tilt angle is often specified by pilot command, or a prior mapping from vehicle velocity [8]. More recent approaches use nonlinear back-stepping controllers [9], but no attention was given to the aerodynamics. The traditional method employs controllers for different operating conditions and transitions between them. A robust transition strategy is needed to make sure a vehicle is kept within a desired flight envelope [10], [11]. Some work on tail-sitter VTOLs considers external forces including aerodynamics. In [12], controllers were designed that both store optimal attitude control solutions and solve trajectory planning online. When multicopter control is used along with aerodynamic force compensation [13], reasonable performance of the vehicle is achieved on a tail-sitter, but the method under-utilizes lift generation from wings and relies on specific vehicle design.

As far as multi-dimensional control forces are concerned, fully-actuated multicopters [14]-[18] have garnered much attention in recent years. Because of the added dimension in force space, vehicle can generate force in any direction without changing attitude [17], [18]. If this is not achieved, then desired attitude is found via the closest projection of 


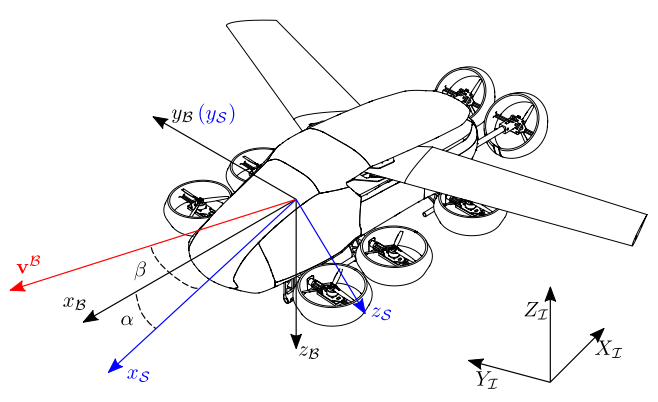

Fig. 2. Illustration of an example VTOL aircraft and associated frames of references: (1) inertial Frame $\mathcal{I}$; (2) body frame $\mathcal{B}$; stability frame $\mathcal{S}$.

desired force onto force space [15], [16]. However, all of such work does not consider aerodynamic forces and only focuses on the concept of full-actuation which attempts to marginalize attitude determination [17]. Aircraft with thrust vectoring have been around for decades, but they have only found applications in military or been used for moment generation during post-stall maneuvers [19]. To the best of our knowledge, no prior work has been done on jointly considering aerodynamic and multi-dimensional input forces, and we intend to fill this gap.

Contribution and Organization. In this paper, we consider a general winged VTOL that has both active aerodynamic elements providing significant forces, as well as multidimensional thrust generation. In Sec. II, a unified definition of a winged VTOL vehicle control problem is presented along with a rigid body and aerodynamics model used for modeling and control design. Sec. III starts with robust position and attitude tracking controllers using forces and moments as inputs, and then a force allocation method that realizes desired forces is presented. In Sec. IV, a strategy for thruster control allocation is presented. The proposed method can generate desired forces and moments while avoiding control saturation. Finally in Sec. V, simulation and experimental results using a prototype winged VTOL are discussed.

\section{SYSTEM DESCRIPTION}

We consider a VTOL vehicle that can produce forces directly with distributed electric propulsion and non-negligible aerodynamic forces and moments from its body or wings. An example VTOL is given in Fig. 2, which is comprised of six side rotors that can produce upward forces, two back rotors that are able to produce forward and upward forces, and a pair of wings used for lift production.

\section{A. General VTOL Vehicle Kinematics and Dynamics}

Consider the vehicle as a six degrees-of-freedom (DoF) rigid body. Define vehicle states as: global position $\mathbf{p}^{\mathcal{I}} \in \mathbb{R}^{3}$ with respect to the inertial frame $\mathcal{I}$; inertial velocity $\mathbf{v}^{\mathcal{B}} \in \mathbb{R}^{3}$ expressed in the body frame $\mathcal{B}$; attitude rotation matrix $R \in$ $S O(3)$; and body-fixed angular rates $\boldsymbol{\omega} \in \mathbb{R}^{3}$. Then vehicle dynamics is given as

$$
\begin{aligned}
\dot{\mathbf{p}}^{\mathcal{I}} & =R \mathbf{v}^{\mathcal{B}}, & m \dot{\mathbf{v}}^{\mathcal{B}} & =m \mathbf{v}^{\mathcal{B}} \times \boldsymbol{\omega}+\mathbf{f}_{\text {ext }}^{\mathcal{B}}, \\
\dot{R} & =R S(\boldsymbol{\omega}), & J \dot{\boldsymbol{\omega}} & =J \boldsymbol{\omega} \times \boldsymbol{\omega}+\boldsymbol{\tau}_{\text {ext }}^{\mathcal{B}},
\end{aligned}
$$

where $m$ denotes the total mass of the vehicle and $J$ is a moment of inertia matrix. Both $m$ and $J$ are considered to be constants. $S(\cdot)$ is a skew-symmetric mapping such that $\mathbf{a} \times \mathbf{b}=S(\mathbf{a}) \mathbf{b}$, and $\mathbf{f}_{\text {ext }}^{\mathcal{B}}$ and $\boldsymbol{\tau}_{\text {ext }}^{\mathcal{B}}$ are the total external force and moment exerted on the vehicle expressed in the body frame. The total external force and moments can be further expanded as

$$
\begin{aligned}
\mathbf{f}_{\text {ext }}^{\mathcal{B}} & =\mathbf{f}_{t}^{\mathcal{B}}\left(\mathbf{u}_{t}, \boldsymbol{\delta}_{c}\right)+\mathbf{f}_{a}^{\mathcal{B}}\left(\mathbf{v}^{\mathcal{B}}, \boldsymbol{\omega}, \boldsymbol{\delta}_{a}\right)+R^{\top} m \mathbf{g}, \\
\boldsymbol{\tau}_{\mathrm{ext}}^{\mathcal{B}} & =\boldsymbol{\tau}_{t}^{\mathcal{B}}\left(\mathbf{u}_{t}, \boldsymbol{\delta}_{c}\right)+\boldsymbol{\tau}_{a}^{\mathcal{B}}\left(\mathbf{v}^{\mathcal{B}}, \boldsymbol{\omega}, \boldsymbol{\delta}_{a}\right),
\end{aligned}
$$

with $\mathbf{g}=[0,0, g]^{\top}$ as a gravity vector. The additional terms $\mathbf{f}_{t}^{\mathcal{B}}$ and $\boldsymbol{\tau}_{t}^{\mathcal{B}}$ denote the combined force and moment generated from distributed propulsion, and $\mathbf{f}_{a}^{\mathcal{B}}$ and $\tau_{a}^{\mathcal{B}}$ are aerodynamic force and moment. The rest are control input vectors for thruster forces $\mathbf{u}_{t} \in \mathbb{R}^{n_{t}}$, deflection angles of control surfaces on wings $\boldsymbol{\delta}_{a} \in \mathbb{R}^{n_{a}}$, and thruster directions $\boldsymbol{\delta}_{c} \in \mathbb{R}^{n_{c}}$.

In general, depending on the configuration of the vehicle and the specific types of thrusters used, there would exist interference between aerodynamic surfaces and thrusters. Those effects are ignored in the present formulation.

\section{B. Thruster Model}

Let the force developed by $i$-th thruster in the body frame be $f_{i} \hat{\mathbf{z}}_{i}$, where $\hat{\mathbf{z}}_{i}$ is the unit vector in the thrust direction. In general, thrusters will also produce a moment around $\hat{\mathbf{z}}_{i}$ as $\tau_{i} \hat{\mathbf{z}}_{i}$, with $\tau_{i}$ proportional to $f_{i}$ by a factor $\gamma_{i}$. For example, force and moment generated by a propeller can be written as $f_{\text {prop }}=C_{T} \rho \nu^{2} d^{4}$ and $\tau_{\text {prop }}=C_{Q} \rho \nu^{2} d^{5}$, where $C_{T}$ and $C_{Q}$ are thrust and moment coefficients, $\rho$ is the density of ambient air, $d$ the diameter of a propeller, and $\nu$ is rotation speed of the propeller. In this case, the coupling factor is $\gamma=\left(C_{Q} d\right) / C_{T}$. Without loss of generality, we can safely assume direct control over each thruster is given.

Each thruster generates a moment with respect to a center of mass of the vehicle by $\boldsymbol{\tau}_{i, \mathrm{~cm}}=f_{i}\left(\mathbf{r}_{i} \times \hat{\mathbf{z}}_{i}+\gamma_{i} \hat{\mathbf{z}}_{i}\right)=f_{i} \boldsymbol{\mu}_{i}$, where $\mathbf{r}_{i}$ is the position of the thruster with respect to the center of mass. Let $\mathbf{u}_{t}=\left[f_{1}, \cdots, f_{n_{t}}\right]^{\top}$ be the thruster force input vector, then

$$
\left[\begin{array}{l}
\mathbf{f}_{t}^{\mathcal{B}}\left(\mathbf{u}_{t}, \boldsymbol{\delta}_{c}\right) \\
\boldsymbol{\tau}_{t}^{\mathcal{B}}\left(\mathbf{u}_{t}, \boldsymbol{\delta}_{c}\right)
\end{array}\right]=\underbrace{\left[\begin{array}{lll}
\hat{\mathbf{z}}_{1}\left(\boldsymbol{\delta}_{c}\right) & \cdots & \hat{\mathbf{z}}_{n_{t}}\left(\boldsymbol{\delta}_{c}\right) \\
\boldsymbol{\mu}_{1}\left(\boldsymbol{\delta}_{c}\right) & \cdots & \boldsymbol{\mu}_{n_{t}}\left(\boldsymbol{\delta}_{c}\right)
\end{array}\right]}_{B_{t}\left(\boldsymbol{\delta}_{c}\right)} \mathbf{u}_{t} .
$$

Through careful design of the vehicle's configuration, one can get a well-conditioned $B_{t}\left(\boldsymbol{\delta}_{c}\right)$ matrix. The process of determining $\mathbf{u}_{t}$ from required force and moment is known as control allocation [20]. More details on this subject are provided in Sec. IV.

\section{Aerodynamic Model}

Assuming no ambient wind, $\mathbf{v}^{\mathcal{B}}=\left[v_{x}, v_{y}, v_{z}\right]^{\top}$ denotes the relative wind velocity. From the body frame $\mathcal{B}$, we first rotate around the negative $y$-axis by an angle $\alpha$ to get the stability frame $\mathcal{S}$, and then around the $z$-axis by $\beta$ to get the wind frame $\mathcal{W}$ (Fig. 2). The angles $\alpha$ and $\beta$ are called angleof-attack and side-slip angle, respectively. Let $V_{\infty}, V_{x z}$, and 

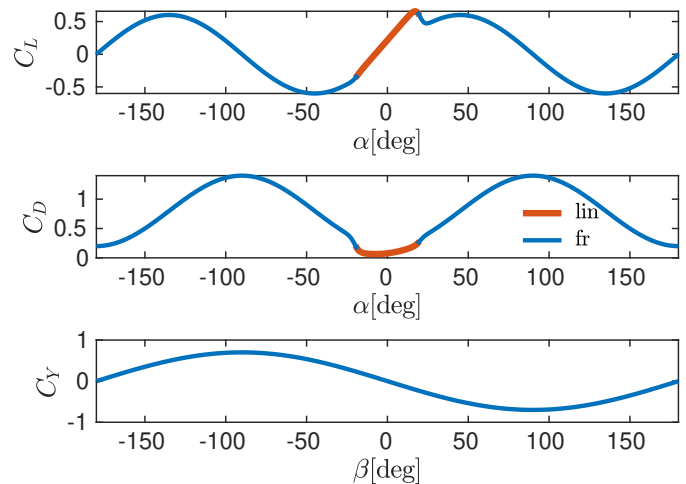

Fig. 3. Demonstration of aerodynamic coefficient from $[-180,180]$ degrees

$V_{x y}$ be free-stream velocity, and projected velocities in body $x z$-plane and $x y$-plane, respectively, which are calculated as,

$$
V_{\infty}=\left\|\mathbf{v}^{\mathcal{B}}\right\|, V_{x z}=\sqrt{v_{x}^{2}+v_{z}^{2}}, \quad V_{x y}=\sqrt{v_{x}^{2}+v_{y}^{2}}
$$

It follows that $\alpha=\operatorname{atan} 2\left(v_{z}, v_{x}\right)$ and $\beta=\arcsin \left(\frac{v_{y}}{V_{\infty}}\right)$.

For modeling simplicity, aerodynamic forces are defined in $\mathcal{S}$ as,

$$
f_{L}=\frac{1}{2} \rho V_{x z}^{2} S_{\mathrm{ref}} C_{L}, f_{D}=\frac{1}{2} \rho V_{x z}^{2} S_{\mathrm{ref}} C_{D}, f_{Y}=\frac{1}{2} \rho V_{x y}^{2} S_{\mathrm{ref}} C_{Y},
$$

where $S_{\text {ref }}$ and $\bar{c}$ denote the pre-selected reference area and reference chord length based on the vehicle geometry [21]. Similarly, the moments in $\mathcal{B}$ are denoted by $\tau_{a, j}=\frac{1}{2} \rho V_{\infty}^{2} S_{\text {ref }} \bar{c} C_{m_{j}}, j=\{x, y, z\}$.

For aircraft, the non-dimensional coefficients $C_{L}, C_{D}, C_{Y}$, $C_{m_{x}}, C_{m_{y}}$, and $C_{m_{z}}$ are typically tabulated from wind tunnel tests as function of various body and aerodynamic states. We use the following method that combines models of different accuracy. The general full range flat-plate model $(\cdot)^{\mathrm{fr}}$ can be used to get the overall trend, while the linear model $(\cdot)^{\text {lin }}$ provides more accuracy at low $\alpha$ :

$$
\begin{array}{ll}
C_{L}^{\mathrm{fr}}=k_{L} \sin (2 \alpha), & C_{L}^{\mathrm{lin}}=C_{L_{0}}+C_{L_{\alpha}} \alpha, \\
C_{D}^{\mathrm{fr}}=k_{D 1} \sin ^{2} \alpha+k_{D 0}, & C_{D}^{\mathrm{lin}}=C_{D_{0}}+k_{C_{L}} C_{L}^{2}, \\
C_{Y}^{\mathrm{fr}}=k_{Y} \sin \beta, & C_{Y}^{\mathrm{lin}}=C_{L_{\beta}} \beta .
\end{array}
$$

We use a hyperbolic tangent tanh blending function to aggregate two models together [22]. The blending of two models can be tuned for accurate prediction within the desired operating range, and bounded error everywhere else. Figure 3 shows the result of this method plot on full ranges of $\alpha$ and $\beta$. Qualitatively, the result demonstrates similar trend compared to test data for both airfoils [23] and real aircraft [13].

On the other hand, the moment coefficients are computed using a linear model refered from [21], assuming a VTOL vehicle is designed to be symmetric with respect to the $x z$ plane. We find

$$
C_{m_{x}}=k_{x_{0}}+k_{x_{1}} \frac{\bar{c} \omega_{x}}{2 V_{\infty}}+k_{x_{2}} \frac{\bar{c} \omega_{z}}{2 V_{\infty}}+k_{x_{3}} \beta
$$

and $C_{m_{y}}$ and $C_{m_{z}}$ are similarly defined. The control surface deflections $\delta_{a}$ are assumed to only affect the aerodynamic

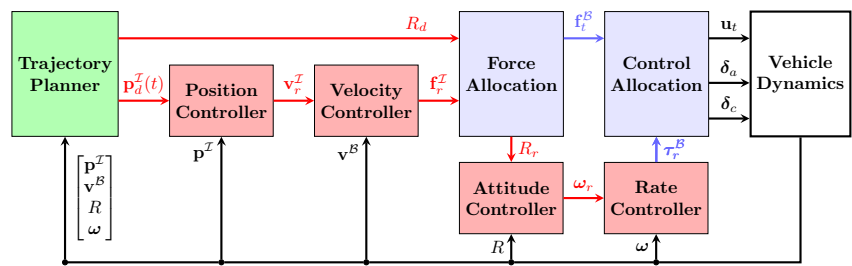

Fig. 4. Schematic of the proposed controller framework for winged VTOL aircraft or flying cars.

moments linearly:

$$
\Delta C_{m_{i}}=\sum_{j=1}^{n_{a}} \bar{b}_{i j} \delta_{j}, \quad i \in\{x, y, z\} .
$$

To summarize, by rotating the aerodynamic forces from $\mathcal{S}$ to $\mathcal{B}$ and decomposing $\tau_{a}^{\mathcal{B}}$ into state-dependent and control affine terms, we have

$$
\mathbf{f}_{a}^{\mathcal{B}}=R_{\mathcal{S}}^{\mathcal{B}}\left[\begin{array}{c}
-f_{D} \\
f_{Y} \\
-f_{L}
\end{array}\right], \quad \boldsymbol{\tau}_{a}^{\mathcal{B}}=\left[\begin{array}{c}
\tau_{m_{x}} \\
\tau_{m_{y}} \\
\tau_{m_{z}}
\end{array}\right]+B_{a} \boldsymbol{\delta}_{a}
$$

with elements of $B_{a}$ derived from (9) as $B_{a, i j}=$ $(1 / 2) \rho V_{\infty}^{2} S_{\text {ref }} \bar{c} \bar{b}_{i j}$.

\section{Controller Design}

By substituting (4) and (10) into (3), we can rewrite $\mathbf{f}_{\text {ext }}^{\mathcal{B}}$ and $\tau_{\text {ext }}^{\mathcal{B}}$ in the following form,

$$
\left[\begin{array}{c}
\mathbf{f}_{\text {ext }}^{\mathcal{B}} \\
\boldsymbol{\tau}_{\text {ext }}^{\mathcal{B}}
\end{array}\right]=\left[\begin{array}{c}
R^{\top} m \mathbf{g} \\
0
\end{array}\right]+\left[\begin{array}{c}
\mathbf{f}_{a}\left(\mathbf{v}^{\mathcal{B}}\right) \\
\boldsymbol{\tau}_{a}\left(\mathbf{v}^{\mathcal{B}}, \boldsymbol{\omega}\right)
\end{array}\right]+\left[\begin{array}{c}
0 \\
B_{a} \boldsymbol{\delta}_{a}
\end{array}\right]+B_{t}\left(\boldsymbol{\delta}_{\boldsymbol{c}}\right) \mathbf{u}_{t} .
$$

We see that $B_{t} \mathbf{u}_{t}$ and $B_{a} \boldsymbol{\delta}_{a}$ indicate that direct inversion can be applied to generate forces and moments, while $\mathbf{f}_{a}$ presents difficulty in determining the desired attitude. We intend to use $\mathbf{f}_{r}^{\mathcal{I}}=R \mathbf{f}_{r}^{\mathcal{B}}$ and $\boldsymbol{\tau}_{r}^{\mathcal{B}}$ and replace $\mathbf{f}_{\text {ext }}^{\mathcal{B}}$ and $\tau_{\text {ext }}^{\mathcal{B}}$ as control inputs in our position and attitude dynamics. Then we focus on deriving a force allocation method that outputs desired attitude that ensures $\mathbf{f}_{\text {ext }}^{\mathcal{B}} \rightarrow R^{\top} \mathbf{f}_{r}^{\mathcal{I}}$. The proposed control system architecture is shown in Fig. 4.

\section{A. Position Tracking Controller}

Given a desired position trajectory $\mathbf{p}_{d}^{\mathcal{I}}(t)$ to track, a position error is defined as $\tilde{\mathbf{p}}=\mathbf{p}^{\mathcal{I}}-\mathbf{p}_{d}^{\mathcal{I}}$. We intend to design a manifold $\mathbf{s}_{v}$ on which the position error converges to zero exponentially:

$$
\mathbf{s}_{v}=\dot{\tilde{\mathbf{p}}}+\Lambda_{p} \tilde{\mathbf{p}}=\mathbf{v}^{\mathcal{I}}-\left(\mathbf{v}_{d}^{\mathcal{I}}-\Lambda_{p} \tilde{\mathbf{p}}\right),
$$

where $\Lambda_{p}$ is a positive definite gain matrix for the position error. We propose the following controller using a required net force $\mathbf{f}_{r}^{\mathcal{I}}$ as an input:

Proposition 1: The position controller is defined as

$$
\begin{gathered}
\mathbf{f}_{r}^{\mathcal{I}}=m \dot{\mathbf{v}}_{r}^{\mathcal{I}}-K_{v} \mathbf{s}_{v}-K_{p} \tilde{\mathbf{p}}, \text { with } \\
\mathbf{s}_{v}=\mathbf{v}^{\mathcal{I}}-\mathbf{v}_{r}^{\mathcal{I}}, \quad \mathbf{v}_{r}^{\mathcal{I}}=\mathbf{v}_{d}^{\mathcal{I}}-\Lambda_{p} \tilde{\mathbf{p}},
\end{gathered}
$$

where $K_{v}$ and $K_{p}$ are positive definite matrices. Suppose the difference between $\mathbf{f}_{r}^{\mathcal{I}}$ and its realization is $\tilde{\mathbf{f}}$. If it is bounded by some $\|\tilde{\mathbf{f}}\| \leq \epsilon$, then $\mathbf{v}^{\mathcal{I}} \rightarrow \mathbf{v}_{r}^{\mathcal{I}}$ and $\mathbf{p}^{\mathcal{I}} \rightarrow \mathbf{p}_{d}^{\mathcal{I}}$ exponentially 
within an ball of radius $r$, denoted as $b_{r}$, controlled by $\epsilon$ and gain matrices $\Lambda_{p}, K_{p}$, and $K_{v}$.

Proof: Using (1), (13) and (14), we get the closed-loop dynamics of $\mathbf{s}_{v}$ :

$$
m \dot{\mathbf{s}}_{v}+K_{v} \mathbf{s}_{v}+K_{p} \tilde{\mathbf{p}}=\tilde{\mathbf{f}}
$$

Differentiating the Lyapunov function $V\left(\mathbf{s}_{v}, \tilde{\mathbf{p}}\right)=$ $(1 / 2)\left(m\left\|\mathbf{s}_{v}\right\|^{2}+\tilde{\mathbf{p}}^{\top} K_{p} \tilde{\mathbf{p}}\right)$ yields

$$
\begin{aligned}
\dot{V} & =\mathbf{s}_{v}^{\top}\left(-K_{v} \mathbf{s}_{v}-K_{p} \tilde{\mathbf{p}}+\tilde{\mathbf{f}}\right)+\tilde{\mathbf{p}}^{\top} K_{p}\left(\mathbf{s}_{v}-\Lambda_{p} \tilde{\mathbf{p}}\right) \\
& =-\mathbf{s}_{v}^{\top} K_{v} \mathbf{s}_{v}-\tilde{\mathbf{p}} K_{p} \Lambda_{p} \tilde{\mathbf{p}}+\mathbf{s}_{v}^{\top} \tilde{\mathbf{f}} .
\end{aligned}
$$

By letting $\zeta=\left[\mathbf{s}_{v}^{\top}, \tilde{\mathbf{p}}^{\top}\right]^{\top}$ and using the comparison method from [24], we get

$$
\|\boldsymbol{\zeta}(t)\| \leq \sqrt{\frac{c_{2}}{c_{1}}}\left\|\boldsymbol{\zeta}\left(t_{0}\right)\right\| \exp \left(\frac{c_{3}}{c_{2}}\left(t-t_{0}\right)\right)+\frac{c_{2}}{c_{1} c_{3}} \sup _{t \geq t_{0}}\|\tilde{\mathbf{f}}\|,
$$

with $c_{1}=\min \left\{m, \lambda_{\min }\left(K_{p}\right)\right\}, c_{2}=\max \left\{m, \lambda_{\max }\left(K_{p}\right)\right\}$, and $c_{3}=\min \left\{\lambda_{\min }\left(K_{v}\right), \lambda_{\min }\left(K_{p}\right) \lambda_{\min }\left(\Lambda_{p}\right)\right\}$. Moreover, $\|\tilde{\mathbf{f}}\| \leq \epsilon$ on a compact set.

$\therefore \sqrt{\left\|\mathbf{s}_{v}\right\|^{2}+\|\tilde{\mathbf{p}}\|^{2}} \rightarrow b_{r}$ with $r=\left(\epsilon c_{2}\right) /\left(c_{1} c_{3}\right)$.

\section{B. Global Attitude Tracking Controller}

We design a controller that provides global tracking of any attitude trajectory. Assume desired time trajectories of attitude $R_{d}(t)$ and its associated angular velocity $\boldsymbol{\omega}_{d}(t)$ are given by the force allocation block, and define an error rotation matrix is defined as $\tilde{R}=R_{d}^{\top} R$. Following [25], it can be shown that the error function used in $S O(3)$ is equivalent to the vector part of the error quaternion $\tilde{\mathbf{q}}=\left[\tilde{q}_{0}, \tilde{\mathbf{q}}_{v}^{\top}\right]^{\top}$ from $\tilde{R}$ with $\tilde{q}_{0} \geq 0$ :

$$
\tilde{q}_{0}=\frac{1}{2} \sqrt{1+\operatorname{tr}(\tilde{R})}, \quad \tilde{\mathbf{q}}_{v}=\frac{1}{4 \tilde{q}_{0}}\left(\tilde{R}-\tilde{R}^{\top}\right)^{\vee},
$$

where the $\vee$ symbol here denotes the vee map which is the inverse operation of $S(\cdot)$. We use singularity-free formulas when $\operatorname{tr}(\tilde{R})=-1$ [26], [27]. The angular rate error $\mathbf{e}_{\omega}$ can be obtained by differentiating $\tilde{R}$ with respect to time as

$$
\dot{\tilde{R}}=R_{d}^{\top} \dot{R}+\dot{R}_{d}^{\top} R=\tilde{R} S\left(\boldsymbol{\omega}-\tilde{R}^{\top} \boldsymbol{\omega}_{d}\right),
$$

which gives $\mathbf{e}_{\omega}=\boldsymbol{\omega}-\tilde{R}^{\top} \boldsymbol{\omega}_{d}$ in the current body frame. The kinematic relation between $\dot{\tilde{\mathbf{q}}}$ and $\mathbf{e}_{\omega}$ is given as

$$
\dot{\tilde{\mathbf{q}}}=\frac{1}{2} W(\tilde{\mathbf{q}}) \mathbf{e}_{\omega}, \quad W(\tilde{\mathbf{q}})=\left[\begin{array}{c}
-\tilde{\mathbf{q}}_{v}^{\top} \\
\tilde{q}_{0} \mathcal{I}+S\left(\tilde{\mathbf{q}}_{v}\right)
\end{array}\right],
$$

with $W(\tilde{\mathbf{q}})^{\top} W(\tilde{\mathbf{q}})=I_{3 \times 3}$. We augment the quaternion attitude error with $\tilde{q}_{0}-1$. Similar to (13), we design a manifold on which attitude error will exponentially converge:

$$
\mathbf{s}_{\omega}=2 W(\tilde{\mathbf{q}})^{\top}\left(\left[\begin{array}{c}
\dot{\tilde{q}}_{0} \\
\dot{\tilde{\mathbf{q}}}
\end{array}\right]+W(\tilde{\mathbf{q}}) \Lambda_{\omega} W(\tilde{\mathbf{q}})^{\top}\left[\begin{array}{c}
\tilde{q}_{0}-1 \\
\tilde{\mathbf{q}}_{v}
\end{array}\right]\right),
$$

where $\Lambda_{\omega}$ is a $3 \times 3$ positive definite gain matrix. Suppose $\Lambda_{\omega}$ has positive eigenvalues $\left\{\lambda_{1}, \lambda_{2}, \lambda_{3}\right\}$, then $W(\tilde{\mathbf{q}}) \Lambda_{\omega} W(\tilde{\mathbf{q}})^{\top}$ will have eigenvalues $\left\{0, \lambda_{1}, \lambda_{2}, \lambda_{3}\right\}$. Since the fourth element of quaternion is redundant, it is expected that $\tilde{\mathbf{q}}_{v} \rightarrow \mathbf{0}$ exponentially. It is also easy to verify that

$$
W(\tilde{\mathbf{q}})^{\top}\left[\begin{array}{c}
\tilde{q}_{0}-1 \\
\tilde{\mathbf{q}}_{v}
\end{array}\right]=\tilde{\mathbf{q}}_{v} .
$$

Our attitude control law is inspired by [28] with an additional back-stepping term.

Proposition 2: Suppose the control law is defined as

$$
\begin{aligned}
\boldsymbol{\tau}_{r}^{\mathcal{B}} & =J \dot{\boldsymbol{\omega}}_{r}-J \boldsymbol{\omega} \times \boldsymbol{\omega}_{r}-K_{\omega} \mathbf{s}_{\omega}-k_{q} \tilde{\mathbf{q}}_{v}, \\
\mathbf{s}_{\omega} & =\boldsymbol{\omega}-\boldsymbol{\omega}_{r}, \quad \boldsymbol{\omega}_{r}=\tilde{R}^{\top} \boldsymbol{\omega}_{d}-2 \Lambda_{\omega} \tilde{\mathbf{q}}_{v} .
\end{aligned}
$$

In the above, $K_{\omega}$ is a positive definite matrix and $k_{q}>0$. Suppose the difference between $\tau_{r}^{\mathcal{B}}$ and its realization is $\tilde{\tau}$. If it is bounded by some $\|\tilde{\boldsymbol{\tau}}\| \leq \epsilon$, then $\tilde{\mathbf{q}}_{v}(t) \rightarrow \mathbf{0}$ and $\boldsymbol{\omega} \rightarrow \boldsymbol{\omega}_{r}$ exponentially within $b_{r}$ controlled by $\epsilon$ and the gain matrices $\Lambda_{q}, k_{q}$, and $K_{\omega}$.

Proof: Select the candidate Lyapunov function as

$$
V\left(\mathbf{s}_{\omega}, \tilde{\mathbf{q}}_{v}\right)=(1 / 2) \mathbf{s}_{\omega}^{\top} J \mathbf{s}_{\omega}+k_{q} \tilde{\mathbf{q}}_{v}^{\top} \tilde{\mathbf{q}}_{v}+k_{q}\left(\tilde{q}_{0}-1\right)^{2} .
$$

$\tilde{q}_{0}=\sqrt{1-\left\|\tilde{\mathbf{q}}_{v}\right\|^{2}}$ is a redundant variable here. Combining dynamics from (2) and controller (20) we get the closed-loop dynamics for $\mathbf{s}_{\omega}$ as

$$
J \dot{\mathbf{s}}_{\omega}+\left(K_{\omega}-S(J \omega)\right) \mathbf{s}_{\omega}+k_{q} \tilde{\mathbf{q}}_{v}=\tilde{\boldsymbol{\tau}}
$$

Using (17), (21) and (22), we compute the time derivative of Lyapunov function as,

$$
\begin{aligned}
\dot{V}= & \mathbf{s}_{\omega}^{\top} J \dot{\mathbf{s}}_{\omega}+2 k_{q} \tilde{\mathbf{q}}_{v}^{\top} \dot{\tilde{\mathbf{q}}}_{v}+2 k_{q}\left(\tilde{q}_{0}-1\right) \dot{\tilde{q}}_{0} \\
= & \mathbf{s}_{\omega}^{\top}\left(S(J \boldsymbol{\omega})-K_{\omega}\right) \mathbf{s}_{\omega}-k_{q} \mathbf{s}_{\omega}^{\top} \tilde{\mathbf{q}}_{v}+\mathbf{s}_{\omega}^{\top} \tilde{\boldsymbol{\tau}} \\
& \quad+k_{q} \tilde{\mathbf{q}}_{v}^{\top}\left(\tilde{q}_{0} I_{3 \times 3}+S\left(\tilde{\mathbf{q}}_{v}\right)\right) \mathbf{e}_{\boldsymbol{\omega}}-k_{q}\left(\tilde{q}_{0}-1\right) \tilde{\mathbf{q}}_{v}^{\top} \mathbf{e}_{\boldsymbol{\omega}} \\
= & -\mathbf{s}_{\omega}^{\top} K_{\omega} \mathbf{s}_{\omega}-2 k_{q} \tilde{\mathbf{q}}_{v}^{\top} \Lambda_{q} \tilde{\mathbf{q}}_{v}+\mathbf{s}_{\omega}^{\top} \tilde{\boldsymbol{\tau}} .
\end{aligned}
$$

By letting $\boldsymbol{\eta}=\left[\mathbf{s}_{\omega}^{\top}, \tilde{\mathbf{q}}_{v}^{\top}\right]^{\top}$ and using the comparison method from [24], we finally get

$$
\|\boldsymbol{\eta}(t)\| \leq \sqrt{\frac{c_{2}}{c_{1}}}\left\|\boldsymbol{\eta}\left(t_{0}\right)\right\| \exp \left(\frac{c_{3}}{c_{2}}\left(t-t_{0}\right)\right)+\frac{c_{2}}{c_{1} c_{3}} \sup _{t \geq t_{0}}\|\tilde{\boldsymbol{\tau}}\|,
$$

with $c_{1}=\min \left\{\lambda_{\min }(J), 2 k_{q}\right\}, c_{2}=\max \left\{\lambda_{\max }(J), 4 k_{q}\right\}$, and $c_{3}=\min \left\{\lambda_{\min }\left(K_{\omega}\right), 2 k_{q} \lambda_{\min }\left(\Lambda_{q}\right)\right\}$. In addition, $\|\tilde{\boldsymbol{\tau}}\| \leq$ $\epsilon$ on a compact set.

$\therefore \quad \sqrt{\left\|\mathbf{s}_{\omega}\right\|^{2}+\left\|\tilde{\mathbf{q}}_{v}\right\|^{2}} \rightarrow b_{r}$ with $r=\left(\epsilon c_{2}\right) /\left(c_{1} c_{3}\right)$.

\section{Force Allocation of VTOL with Wings}

The realization of $\mathbf{f}_{r}^{\mathcal{I}}$ within a small error is essential to minimizing position tracking errors (13). We start by equating $\mathbf{f}_{r}^{\mathcal{I}}=R\left(\mathbf{f}_{t}^{\mathcal{B}}\left(\mathbf{u}_{t}\right)+\mathbf{f}_{a}^{\mathcal{B}}\left(\mathbf{v}^{\mathcal{B}}\right)\right)+m \mathbf{g}$, where the effects of $\boldsymbol{\delta}_{a}$ and $\boldsymbol{\delta}_{c}$ are assumed to be fixed or ignored. Note that $\mathbf{f}_{t}^{\mathcal{B}}$ is directly controlled by fast input dynamics $\mathbf{u}_{t}$ while $\mathbf{f}_{a}^{\mathcal{B}}$ is dependent on both $R$ and $\mathbf{v}^{\mathcal{B}}$. For a forward flying VTOL with wings, we intend to utilize as much wing-lift as possible. Thus $\mathbf{f}_{a}^{\mathcal{B}}$ will be prioritized to reach $\mathbf{f}_{r}^{\mathcal{I}}$, with any residue taken care of by $\mathbf{f}_{t}^{\mathcal{B}}$ :

$$
\mathbf{f}_{t}^{\mathcal{B}}\left(\mathbf{u}_{t}\right)=\mathcal{P}_{\mathcal{F}_{t}}\left(R^{\top}\left(\mathbf{f}_{r}^{\mathcal{I}}-m \mathbf{g}\right)-\mathbf{f}_{a}^{\mathcal{B}}\left(\mathbf{v}^{\mathcal{B}}\right)\right) .
$$

Here, $\mathcal{P}_{\mathcal{F}_{t}}(\cdot)$ is the projection of a force vector onto a feasible force space $\mathcal{F}_{t}$, which is further explained in Sec. IV.

We propose two strategies for fast and slow flight speeds.

Proposition 3 (Low-Speed Force Allocation): Suppose

$\hat{\mathbf{k}}_{c}$ is a unit vector in the body frame indicating the favorable thruster force direction within $\mathcal{F}_{t}$. Then we let

$$
\mathbf{f}_{t}^{\mathcal{B}}\left(\mathbf{u}_{t}\right)=\left(R^{\top}\left(\mathbf{f}_{r}^{\mathcal{I}}-m \mathbf{g}\right)-\mathbf{f}_{a}^{\mathcal{B}}\left(\mathbf{v}^{\mathcal{B}}\right)\right) \cdot \hat{\mathbf{k}}_{c}
$$


as the current thruster force output. $R_{d}$ is obtained as any rotation that satisfies

$$
R_{d} \hat{\mathbf{k}}_{c}=\mathbf{f}_{r}^{\mathcal{I}} /\left\|\mathbf{f}_{r}^{\mathcal{I}}\right\|
$$

To fully determine $R_{d}$, we can constrain $\hat{x}_{\mathcal{B}}$ to be in certain pre-defined heading direction when projected onto $X Y$-plane in $\mathcal{I}$ [29], [30].

Proposition 4 (High-Speed Force Allocation): For highspeed flight, we limit $\beta=0$ and $\alpha$ to be small within a linear region of the aerodynamic model. The desired wind frame $\mathcal{W}$ axes in the current body frame $\mathcal{B}$ is

$$
\hat{x}_{\mathcal{W}}=\frac{\mathbf{v}^{\mathcal{B}}}{\left\|\mathbf{v}^{\mathcal{B}}\right\|}, \quad \hat{y}_{\mathcal{W}}=\frac{\mathbf{v}^{\mathcal{B}} \times \mathbf{f}_{r}^{\mathcal{B}}}{\left\|\mathbf{v}^{\mathcal{B}} \times \mathbf{f}_{r}^{\mathcal{B}}\right\|}, \quad \hat{z}_{\mathcal{W}}=\hat{x}_{\mathcal{W}} \times \hat{y}_{\mathcal{W}} .
$$

The rotation from the body frame to the wind frame is

$$
R_{\mathcal{W}}^{\mathcal{B}}=\left[\hat{x}_{\mathcal{W}}, \hat{y}_{\mathcal{W}}, \hat{z}_{\mathcal{W}}\right],
$$

and $\mathbf{f}_{r}^{\mathcal{W}}$ lies entirely in the $x_{\mathcal{W}} z_{\mathcal{W}}$-plane. Let $f_{\|}=f_{r, x}^{\mathcal{W}}$, $f_{\perp}=f_{r, z}^{\mathcal{W}}$ denote the parallel and perpendicular forces in $\mathcal{W}$. For small $\alpha_{d}$, the balance of forces is simplified as $f_{\|}=f_{t, x}-f_{D}, \quad f_{\perp}=f_{t, z}-f_{L}$. Using (7), we limit the maximum lift allowable by wings as $f_{L \text {, max }}$, given the current velocity. Then the force allocation follows

$$
\left\{\begin{array}{ll}
f_{t, z}=f_{L, \max }+f_{\perp} & \text { if }-f_{\perp}>f_{L, \max } \\
f_{t, z}=0 & \text { if }-f_{\perp} \leq f_{L, \text { max }}
\end{array} .\right.
$$

The desired lift force is simply $f_{L}=f_{t, z}-f_{\perp}$, and we get $\alpha_{d}$ from (6) and (7). Let $R_{\mathcal{D}}^{\mathcal{W}}$ be a rotation around $\hat{y}_{\mathcal{W}}$ by $\alpha_{d}$. The desired attitude is simply

$$
R_{d}=R R_{\mathcal{W}}^{\mathcal{B}} R_{\mathcal{D}}^{\mathcal{W}}
$$

where $R$ is the rotation matrix from $\mathcal{I}$ to $\mathcal{B}$ used in (2). Before proving the boundedness of the force error $\tilde{\mathbf{f}}$, we state the following assumptions:

Assumption 1: The coefficients $C_{L}, C_{Y}$, and $C_{D}$ from (7) admit a lower bounded error $\epsilon_{1}$ on a linear model than $\epsilon_{2}$ on a full-range model.

Assumption 2: Vehicle contained entirely in its flight envelope. The attitude error satisfies $\left\|\tilde{\mathbf{q}}_{v}\right\| \leq \sigma_{1}$ globally. Within linear aerodynamic region, $\left\|\tilde{\mathbf{q}}_{v}\right\| \leq \sigma_{1}<\sigma_{1}$ determined by cruise condition.

Assumption 3: The total thruster force $\mathbf{f}_{t}^{\mathcal{B}}$ can be achieved arbitrarily close to a desired value.

Theorem 3.1: Suppose we combine the desired attitude $R_{d}$ and fed into the attitude controller in Sec. III-B. In quaternion form, thi $\mathbf{q}_{1}$ and $\mathbf{q}_{2}$, from (24) and (28) using $\mathbf{q}_{d}=$ $\operatorname{Slerp}\left(\mathbf{q}_{1}, \mathbf{q}_{2} ; \gamma\right)$, am interpolation scheme for quaternions defined in [31] with a parameter $\gamma$ as a tanh mixing function varying with $V_{\infty}$ and transition around a threshold speed $V_{\text {tr }}$. Assuming $V_{\max }$ is the maximum vehicle speed associated in the trajectory, the overall force allocation guarantees a bound on $\tilde{\mathbf{f}}$ controlled by $\max \left\{2 V_{\text {tr }}^{2} \epsilon_{2}, V_{\max }^{2} \epsilon_{1}\right\}$

Proof: From (23) and (24), denote $\mathbf{f}_{d}=\left(\mathbf{f}_{r}^{\mathcal{I}}-m \mathbf{g}\right)-$ $R \mathbf{f}_{a}^{\mathcal{B}}\left(\mathbf{v}^{\mathcal{B}}\right)$ and $\hat{\mathbf{f}}_{d}$ as the estimated value based on approximate aerodynamics (7). Using assumptions 1 and 2, it can be shown

$$
\begin{aligned}
\|\tilde{\mathbf{f}}\| & \leq\left\|\mathbf{f}_{d}-\mathcal{P}_{\hat{\mathbf{k}}_{c}}\left(\hat{\mathbf{f}}_{d}\right)\right\| \leq\left\|\hat{\mathbf{f}}_{d}-\mathcal{P}_{\hat{\mathbf{k}}_{c}}\left(\hat{\mathbf{f}}_{d}\right)\right\|+\frac{\rho S_{\text {ref }}}{2} V_{\infty}^{2} \epsilon_{2} \\
& \leq \epsilon_{3}\left\|\hat{\mathbf{f}}_{d}\right\|+\frac{\rho S_{\text {ref }}}{2} V_{\infty}^{2} \epsilon_{2},
\end{aligned}
$$

where $\epsilon_{3}<1$ is determined by the global attitude error bound from assumption 2. Similarly from (28), we have

$$
\|\tilde{\mathbf{f}}\| \leq \epsilon_{4}\left\|\hat{\mathbf{f}}_{d}\right\|+\frac{\rho S_{\text {ref }}}{2} V_{\infty}^{2} \epsilon_{1},
$$

where $\epsilon_{4}<\epsilon_{3}$ is satisfied from small angle approximation and $\epsilon_{4} \ll 1$. During transitioning phase when $0<\gamma<1$,

$$
\|\tilde{\mathbf{f}}\| \leq \epsilon_{3}\left\|\hat{\mathbf{f}}_{d}\right\|+\rho S_{\text {ref }} V_{\text {tr }}^{2} \epsilon_{2} .
$$

There for the supreme is bounded by

$$
\sup _{t \geq t_{0}}\|\tilde{\mathbf{f}}\| \leq \epsilon_{3}\left\|\hat{\mathbf{f}}_{d}\right\|+\frac{\rho S_{\text {ref }}}{2} \max \left\{2 V_{\mathrm{tr}}^{2} \epsilon_{2}, V_{\max }^{2} \epsilon_{1}\right\} .
$$

It is interesting to note the intuition behind this bound. $\epsilon_{3}\left\|\hat{\mathbf{f}}_{d}\right\|$ is limited by desired force and attitude respectively, which is achieved internally inside the controller. The first term in $\max \{\cdot\}$ represents a bound with the full-range aerodynamic model having larger uncertainties, limited by $V_{\text {tr }}$. This is a common implicit assumption among multicopter control. The second term in $\max \{\cdot\}$ represents a tighter bound from high accuracy linear model.

\section{Distributed Propulsion Control Allocation}

By fixing $\boldsymbol{\delta}_{c}$ for a mapping $B_{t}\left(\boldsymbol{\delta}_{c}\right) \in \mathbb{R}^{n_{w} \times n_{t}}$, we rewrite (4) with $\mathbf{w}_{t}^{\mathcal{B}}=\left[\left(\mathbf{f}_{t}^{\mathcal{B}}\right)^{\top},\left(\boldsymbol{\tau}_{t}^{\mathcal{B}}\right)^{\top}\right]^{\top} \in \mathbb{R}^{n_{w}}$. Given a feasible control space $\mathcal{U}_{t} \subset \mathbb{R}^{n_{t}}$ of the distributed propulsion, control allocation aims to find $\mathbf{u}_{t} \in \mathcal{U}_{t}$. However, [32] showed there exists no single inverse mapping that can recover a full set of feasible $\mathbf{w}_{t}^{\mathcal{B}}$ without violating some control constraints for an over-actuated system when $n_{t}>n_{w}$. Nonetheless, note that $B_{t}$ has multiple right inverses. Hence, there may still exist more than one $\mathbf{u}_{t}$ satisfying $B_{t} \mathbf{u}_{t}=\mathbf{w}_{t}^{\mathcal{B}}$ with $\mathbf{u}_{t} \in \mathcal{U}_{t}$.

Because control allocation in practice is executed with a very high frequency, we would like to validate if there exists a feasible $\mathbf{u}_{t} \in \mathcal{U}_{t}$ given $\mathbf{w}_{t}^{\mathcal{B}}$ within the same time-scale. We achieve this by pre-computing a full attainable wrench space $\mathcal{W}_{t} \subset \mathbb{R}^{n_{w}}$ for all elements in which it is guaranteed to find $\mathbf{u}_{t} \in \mathcal{U}_{t}$ under some inverse mapping of $B_{t}$. If $\mathbf{w}_{t}^{\mathcal{B}} \in \mathcal{W}_{t}$, then a method proposed in Sect. IV-B is used to solve for $\mathbf{u}_{t}$. Otherwise, the magnitude of $\mathbf{w}_{t}^{\mathcal{B}}$ may have to be scaled down until it becomes an element of $\mathcal{W}_{t}$ with its direction maintained, as is commonly done in the literature [32].

\section{A. Attainable Thruster Wrench Space}

Let $\mathcal{U}_{t}=\left\{\mathbf{u}_{t} \mid \mathbf{u}_{t, \text { min }} \leq \mathbf{u}_{t} \leq \mathbf{u}_{t, \max }\right\}$ and its boundary $\partial \mathcal{U}_{t}=\left\{\mathbf{u}_{t, \min }, \mathbf{u}_{t, \max }\right\}$. A feasible wrench $\mathbf{w}_{t}^{\mathcal{B}}$ lies under the column space of $B_{t}$ with $\mathbf{u}_{t} \in \mathcal{U}_{t}$. We define the attainable wrench space as $\mathcal{W}_{t}=\left\{\mathbf{w}_{t}^{\mathcal{B}}=\sum_{j=1}^{n} u_{t, j} \mathbf{b}_{j} \mid u_{t, \text { min }} \leq u_{t, j} \leq u_{t, \max }, \forall j\right\}$, where $u_{t, j}$ and $\mathbf{b}_{j}$ represent the $j$-th element and column of $\mathbf{u}_{t}$ and $B_{t}$, respectively. Without loss of generality, let us 
assume individual thrusters are identical, with $u_{t, \min }=0$ and $u_{t, \max }=1$. Then, by definition

$$
\mathcal{W}_{t}=\operatorname{Conv}\left(\bigoplus_{j=1}^{n}\left\{\mathbf{0}, \mathbf{b}_{j}\right\}\right)
$$

where $\operatorname{Conv}(\cdot)$ represents a convex hull of a given set and $\bigoplus$ is the Minkowski sum. Checking whether $\mathbf{w}_{t}^{\mathcal{B}} \in \mathcal{W}_{t}$ can be done via linear programming [33]. Even though linear programs run reasonably fast on modern computers, we argue that for many low-power control units on aerial vehicles, the method still poses difficulty, especially when $m$ is large. In such a case, we propose $\mathcal{W}_{t}$ may be approximated by a hypercuboid $\mathcal{W}_{t, \text { app }}$ that is maximally fitted within $\mathcal{W}_{t}$

$$
\mathcal{W}_{t, \text { app }}=\left\{\mathbf{w}_{t}^{\mathcal{B}} \mid \mathbf{w}_{t, \text { min }}^{\mathcal{B}} \leq \mathbf{w}_{t}^{\mathcal{B}} \leq \mathbf{w}_{t, \max }^{\mathcal{B}},\right\} \subset \mathcal{W}_{t},
$$

$\mathbf{w}_{t, \min }^{\mathcal{B}}$ and $\mathbf{w}_{t, \max }^{\mathcal{B}}$ are constant defining boundary of hypercuboid. Although there is a potential partial loss of space in $\mathcal{W}_{t}$, checking $\mathbf{w}_{t}^{\mathcal{B}} \in \mathcal{W}_{t \text {,app }}$ will be much faster than that of $\mathbf{w}_{t}^{\mathcal{B}} \in \mathcal{W}_{t}$, involving only a finite number of comparison operations.

\section{B. Distributed Propulsion Control Allocation}

A right pseudoinverse $B_{t}^{+}=B_{t}^{\top}\left(B_{t} B_{t}^{\top}\right)^{-1}$ of $B_{t}$ is a common saturation-prune method for control allocation often considered in the literature and practice. A straightforward alternative is to solve an optimization,

$$
\begin{array}{cl}
\min _{\mathbf{u}_{t}} & \left\|\mathbf{u}_{t}\right\|_{2}, \\
\text { subject to } & B_{t} \mathbf{u}_{t}=\mathbf{w}_{t}^{\mathcal{B}}, \\
& \mathbf{u}_{t, \min } \leq \mathbf{u}_{t} \leq \mathbf{u}_{t, \max }
\end{array}
$$

which we deemed it not ideal for a real-time application for the abovementioned practical reasons. Note that $\mathbf{u}_{t}$ computed with the right pseudoinverse is a solution to (32) if the inequality constraint is ignored. Therefore, the method of cascading inverse [34] can be promising in computing $\mathbf{u}_{t} \in \mathcal{U}_{t}$, which computes pseudoinverse or generalized inverse solutions in an iterative manner.

For flying aircraft with distributed propulsion, a major portion of control effort goes to lift generation to counterbalance the weight. Based on this observation, we propose an allocation method that computes an inverse mapping $B_{t}^{\prime}$ of $B_{t}$ that avoids saturation of $\mathbf{u}_{t}$ by evenly distributing the effort to generate lift across the thrusters. Furthermore, the allocation prevents thrusters from generating downward forces. The resultant $B_{t}^{\prime}$ can be implemented onboard as a static mapping and its execution speed would be much faster than any online optimization method.

By definition, $B_{t}^{\prime} \mathbf{w}_{t}^{\mathcal{B}}=\mathbf{u}_{t}$. The energy consumption, $\left\|\mathbf{u}_{t}\right\|_{2}^{2}=\left(\mathbf{w}_{t}^{\mathcal{B}}\right)^{\top}\left(B_{t}^{\prime}\right)^{\top} B_{t}^{\prime} \mathbf{w}_{t}^{\mathcal{B}}$, is minimized for a given feasible $\mathbf{w}_{t}^{\mathcal{B}}$ by minimizing the Frobenius norm of $B_{t}^{\prime}$,

$$
\left\|B_{t}^{\prime}\right\|_{F}=\sqrt{\operatorname{tr}\left(B_{t}^{\prime \top} B_{t}^{\prime}\right)}
$$

The following optimization is formulated, which not only minimizes the energy consumption but also guarantees evenly
TABLE I

Physical Parameters for Prototype Winged VTOL

\begin{tabular}{c|ccccc}
$m[\mathrm{~kg}]$ & $J_{x x}$ & $J_{y y}$ & $J_{z z}$ & $J_{x z}$ & {$\left[\mathrm{~kg} \mathrm{~m}^{2}\right]$} \\
\hline 5.5 & 0.134 & 0.252 & 0.346 & -0.004 & \\
\hline
\end{tabular}

TABLE II

Aerodynamic Parameters For Prototype Winged VTOL

\begin{tabular}{cccccc}
$C_{T}$ & $C_{Q}$ & $C_{L_{0}}$ & $C_{L_{\alpha}}\left[\mathrm{rad}^{-1}\right]$ & $C_{D_{0}}$ & $k_{C_{L}}$ \\
\hline 0.182 & 0.0143 & 0.216 & 1.622 & 0.0651 & 0.273 \\
\hline
\end{tabular}

distributed lift generation from all thrusters:

$$
\begin{array}{ll}
\min _{B_{t}^{\prime}} & \left\|B_{t}^{\prime}\right\|_{F}+f_{\mathrm{aad}}\left(\mathbf{b}^{\prime}\right), \\
\text { subject to } & B_{t} B_{t}^{\prime}=I, \\
& \mathbf{b}^{\prime} \leq \mathbf{0},
\end{array}
$$

where $\mathbf{b}^{\prime}$ corresponds to the column of $B_{t}^{\prime}$ governing the allocation of a vertical force. Note that the positive body $z$-direction is downwards in Fig. 2, and to generate an upward vertical lift, it is desirable to have all elements of $\mathbf{b}^{\prime}$ less than zero. In addition, to avoid any individual rotor reaching maximum speed, we distribute the vertical force evenly across thrusters. In particular, the difference between individual thruster forces are minimized using the average absolute deviation $f_{\text {aad }}\left(\mathbf{b}^{\prime}\right)$ about the mean of $\mathbf{b}^{\prime}$, where

$$
f_{\text {aad }}\left(\mathbf{b}^{\prime}\right)=\frac{1}{n_{t}} \sum_{i=1}^{n_{t}}\left|b_{i}^{\prime}-\operatorname{mean}\left(\mathbf{b}^{\prime}\right)\right| .
$$

Note that (33) is convex and can be easily solved through a convex optimization solver.

\section{NUMERICAL AND EXPERIMENTAL RESULTS}

\section{A. Simulation for Take-off, Transition, and Cruise Flight}

We model a vehicle similar to Fig. 2 to demonstrate a typical take-off and transition to cruise behavior. The physical properties listed in Table I are based on the prototype vehicle we have constructed shown in Fig. 1, with $J_{x y} \approx J_{y z} \approx 0$ due to symmetry. Important aerodynamic properties are listed in Table II. $C_{T}$ and $C_{Q}$ are experimentally obtained for a sixinch propeller. The aerodynamic coefficients are calculated through combining both wind-tunnel test data as well as theoretical values of a finite wing and blunt body. Additional coefficients used in (7) and (8) are extracted and adapted from [23], [35].

Given crude velocity command without any sophisticated trajectory design. Figure 5a shows the vehicle's longitudinal states trajectory. Started from rest, the vehicle is commanded with a positive climbing speed $v_{z d}^{\mathcal{I}}=5 \mathrm{~m} / \mathrm{s}$ from $t=0 \mathrm{~s}$ to $t=5 \mathrm{~s}$. Starting at $t=5 \mathrm{~s}$, a forward cruising speed $v_{x d}^{\mathcal{I}}=$ $15 \mathrm{~m} / \mathrm{s}$ is given. Lift, drag, and angle-of-attack are shown in Fig. 5b. The transitional behavior of the vehicle can be clearly seen. During slow climbing stage, the force allocator will use upward force from vertical thrusters. Starting forward flight, the vehicle first pitch forward as a conventional multicopter. 

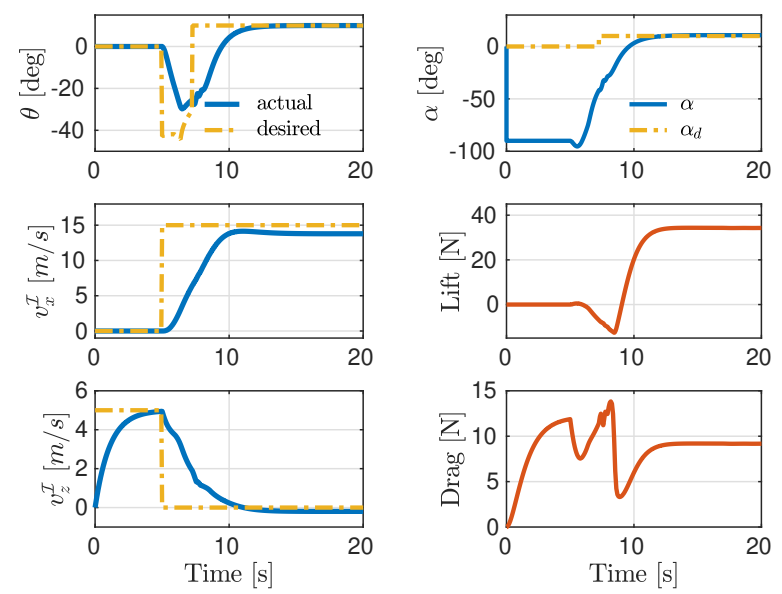

(a) Velocity and Pitch

(b) $\alpha$ and Aerodynamic Forces

Fig. 5. State and commands related to a transition-to-cruise trajectory

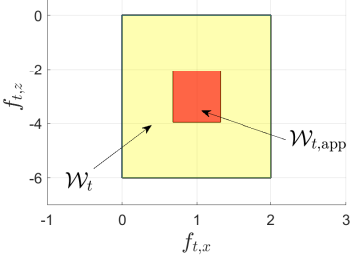

(a) $2 \mathrm{D}$ force space $\mathcal{F}_{t}$

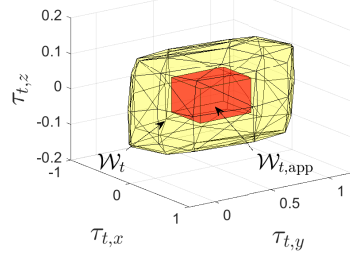

(b) $3 \mathrm{D}$ moment space $\mathcal{M}_{t}$
Fig. 6. Projections of the attainable wrench space $\mathcal{W}_{t}$ and its approximation $\mathcal{W}_{t, \text { app }}$ of the vehicle in Fig. 2 onto (a) 2D force space and (b) 3D moment space. The force space is two-dimensional because the vehicle does not have an ability to generate thrust in its $y$-axis.

After gaining some forward speed, the controller uses positive lift production from wings during fast forward flight.

\section{B. Numerical Simulation of Thruster Control Allocation}

Figure 6 shows five-dimensional spaces $\mathcal{W}_{t}$ and $\mathcal{W}_{t \text {,app }}$ of the vehicle in Fig. 2 projected onto a two-dimensional force space and three-dimensional moment space for visualization. Note that the vehicle does not have an ability to generate a thrust in its $y$-axis, hence the force space is only two-dimensional. A total of $100,000 \mathbf{w}_{t}^{\mathcal{B}}$ from $\mathcal{W}_{t, \text { app }}$ (ten equidistant points along each axis) and computed their associated $\mathbf{u}_{t}$ using the conventional pseudoinverse approach, cascading inverse, and optimization (32). While the conventional pseudoinverse approach returned $\mathbf{u}_{t} \notin \mathcal{U}_{t}$ for $10.8 \%$ of the sampled wrenches, the cascading inverse method returned no saturated control commands for all the test cases, with only about $20 \%$ increase in average computation time. The optimization also returned no $\mathbf{u}_{t} \notin \mathcal{U}_{t}$ but spent much longer time to find solutions. In addition, the costs of $\mathbf{u}_{t}$ obtained by the cascading inverse were nearly the same as the optimal costs obtained by solving (32).

\section{Closed-Loop Flight Experiments}

We implemented our controllers and force allocator from Sec. III, as well as part of the control allocation scheme in Sec. IV, using modified PX4 firmware and Pixhawk flight

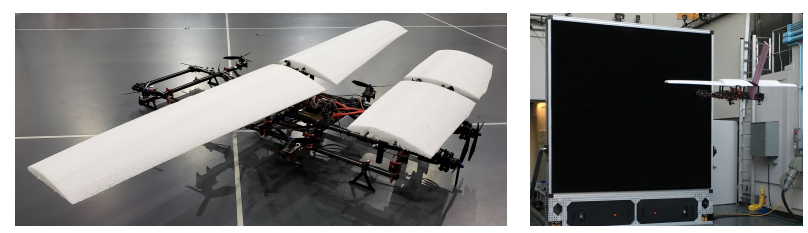

(a) Prototype VTOL with wing and tilt-rotor (b) Fan-array wind tunnel

Fig. 7. Functional prototype winged VTOL flying in wind field generated by a fan-array wind tunnel, provided by Dr. Mory Gharib's group
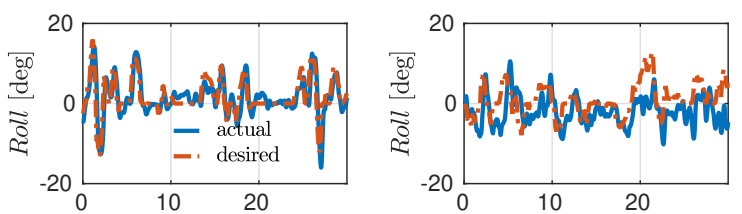

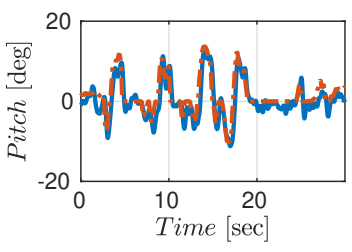

(a) Attitude without wind

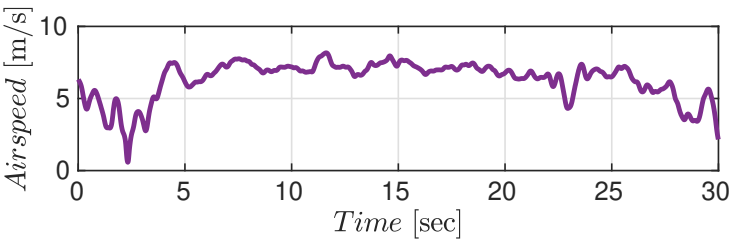

(c) Measured airspeed from onboard pitot-tube during fan-array test

Fig. 8. State and commands related to a transition-to-cruise trajectory

controller [36] on our functional prototype shown in Fig. 7a. The prototype has the same configuration in terms of thrusters and wing compared to Fig. 1. Tests on attitude tracking performance were run at Caltech CAST Flight Arena. We conducted experiments both in normal free-flight and with uniform wind field generated by a fan-array wind tunnel, shown in Fig. $7 b$.

The vehicle has asymmetric rotor placement and aerodynamic surfaces of significant size. Even during normal free flight, we observed noticeable non-vanishing disturbances from wing, ground, and rotor interactions. In spite of that, our proposed controller were able to swiftly track attitude trajectory given by an operator, as shown in Fig. 8a. During fan-array wind tunnel test shown in Fig. 7b, the vehicle is switched to a forward flying mode with tilted back rotor. Tracking error increased due to a larger wind disturbance, but the controller still maintained stability. The onboard pitot-tube measured an airspeed up to $8 \mathrm{~m} / \mathrm{s}$.

\section{CONCLUSION AND Future WORK}

We presented a novel framework for designing controllers for a VTOL aircraft with wing. The control design objective is split into: (a) designing nonlinear position/velocity and attitude/rate controllers using net forces and moments as input; and (b) force, moment, and control allocations to 
generate the desired wrench. We identified the problem of force allocation is complex and vital to a winged VTOL aircraft under substantial aerodynamic forces, and proposed a general solution for closed-loop flight in varying speed regimes. Furthermore, the attainable force, moments, and control spaces are analyzed to give a real-time verifiable set to avoid control saturation. An prototype hybrid VTOL vehicle is constructed. Realistic data are used to model and simulate the behavior of the proposed framework in commanding a transitional maneuver of the vehicle without careful trajectory design. Experiments were carried out to demonstrate the stability and robustness of the proposed controller and allocation design. Future work includes further generalization of the method to apply to different hybrid VTOL models flying in a real-world environment.

\section{ACKNOWLEDGEMENT}

The authors thank M. Gharib, Director of CAST, for his support and technical guidance. The authors also thank R. Nemovi, M. Veismann, E. Yu, C. Dougherty, and J. Burdick at Caltech, and P. Tokumaru at AeroVironment.

\section{REFERENCES}

[1] J. Holden and N. Goel, "Uber elevate: Fast-forwarding to a future of on-demand urban air transportation," Uber, Tech. Rep., October 2016.

[2] P. Menon, M. Badgett, R. Walker, and E. Duke, "Nonlinear flight test trajectory controllers for aircraft," J. Guid. Control, Dyn., vol. 10, no. 1, pp. 67-72, 1987.

[3] M.-D. Hua, T. Hamel, P. Morin, and C. Samson, "A control approach for thrust-propelled underactuated vehicles and its application to vtol drones," IEEE Trans. Autom. Control, vol. 54, no. 8, pp. 1837-1853, 2009.

[4] M. D. Moore, "Distributed electric propulsion (DEP) aircraft," NASA Langley Research Center," , 2012.

[5] A. M. Stoll, J. Bevirt, M. D. Moore, W. J. Fredericks, and N. K. Borer, "Drag reduction through distributed electric propulsion," in 14th AIAA Aviation Tech., Integration Oper. Conf., 2014, p. 2851.

[6] C. Papachristos, K. Alexis, G. Nikolakopoulos, and A. Tzes, "Model predictive attitude control of an unmanned tilt-rotor aircraft," in IEEE Int. Symp. on Ind. Elec., 2011, pp. 922-927.

[7] R. T. Rysdyk and A. J. Calise, "Adaptive model inversion flight control for tilt-rotor aircraft," J. Guid. Control, Dyn., vol. 22, no. 3, pp. 402407, 1999.

[8] S. Park, J. Bae, Y. Kim, and S. Kim, "Fault tolerant flight control system for the tilt-rotor uav," J. Franklin Institute, vol. 350, no. 9, pp. 2535-2559, 2013.

[9] A. B. Chowdhury, A. Kulhare, and G. Raina, "Back-stepping control strategy for stabilization of a tilt-rotor uav," in Chinese Control Dec. Conf., 2012, pp. 3475-3480.

[10] A. Frank, J. McGrew, M. Valenti, D. Levine, and J. How, "Hover, transition, and level flight control design for a single-propeller indoor airplane," in AIAA Guid. Nav. Control Conf., 2007, p. 6318.

[11] R. H. Stone, P. Anderson, C. Hutchison, A. Tsai, P. Gibbens, and K. Wong, "Flight testing of the t-wing tail-sitter unmanned air vehicle," J. Aircraft, vol. 45, no. 2, pp. 673-685, 2008.

[12] R. Ritz and R. D'Andrea, "A global controller for flying wing tailsitter vehicles," in IEEE Int. Conf. Robotics Autom. (ICRA), 2017, pp. 27312738.

[13] A. Oosedo, S. Abiko, A. Konno, and M. Uchiyama, "Optimal transition from hovering to level-flight of a quadrotor tail-sitter uav," Autonomous Robots, vol. 41, no. 5, pp. 1143-1159, 2017.

[14] S. Rajappa, M. Ryll, H. H. Bülthoff, and A. Franchi, "Modeling, control and design optimization for a fully-actuated hexarotor aerial vehicle with tilted propellers," in IEEE Int. Conf. Robotics Autom. (ICRA), 2015, pp. 4006-4013.

[15] M. Ryll, D. Bicego, and A. Franchi, "Modeling and control of fast-hex: a fully-actuated by synchronized-tilting hexarotor," in IEEE/RSJ Int. Conf. Intelli. Robots Sys. (IROS) 2016, 2016.
[16] A. Franchi, R. Carli, D. Bicego, and M. Ryll, "Full-pose tracking control for aerial robotic systems with laterally bounded input force," IEEE Trans. Robotics, vol. 34, no. 2, pp. 534-541, 2018.

[17] D. Brescianini and R. D'Andrea, "Design, modeling and control of an omni-directional aerial vehicle," in IEEE Int. Conf. Robotics Autom. (ICRA). IEEE, 2016, pp. 3261-3266.

[18] M. Kamel, S. Verling, O. Elkhatib, C. Sprecher, P. Wulkop, Z. Taylor, R. Siegwart, and I. Gilitschenski, "Voliro: An omnidirectional hexacopter with tiltable rotors," arXiv preprint arXiv:1801.04581, 2018.

[19] S. A. Snell, D. F. Nns, and W. L. Arrard, "Nonlinear inversion flight control for a supermaneuverable aircraft," J. Guid. Control, Dyn., vol. 15, no. 4, pp. 976-984, 1992.

[20] T. A. Johansen and T. I. Fossen, "Control allocation a survey," Automatica, vol. 49, no. 5, pp. 1087-1103, 2013.

[21] B. Etkin, Dynamics of atmospheric flight. Courier Corporation, 2012.

[22] C. Rumsey and V. Vatsa, "A comparison of the predictive capabilities of several turbulence models using upwind and central-difference computer codes," in 31st Aerospace Sciences Meeting, 1993, p. 192.

[23] R. E. Sheldahl and P. C. Klimas, "Aerodynamic characteristics of seven symmetrical airfoil sections through 180-degree angle of attack for use in aerodynamic analysis of vertical axis wind turbines," Sandia National Labs., Albuquerque, NM (USA), Tech. Rep., 1981.

[24] H. Khalil, Nonlinear Systems. Prentice Hall, 2002.

[25] T. Lee, "Exponential stability of an attitude tracking control system on $\mathrm{SO}(3)$ for large-angle rotational maneuvers," Systems \& Control Letters, vol. 61, no. 1, pp. 231-237, 2012.

[26] S. W. Shepperd, "Quaternion from rotation matrix.[four-parameter representation of coordinate transformation matrix]," J.Guid. Control, vol. 1, pp. 223-224, 1978.

[27] A. R. Klumpp, "Singularity-free extraction of a quaternion from a direction-cosine matrix," J. Spacecraft Rockets, vol. 13, no. 12, pp. 754-755, 1976.

[28] S. Bandyopadhyay, S.-J. Chung, and F. Y. Hadaegh, "Nonlinear attitude control of spacecraft with a large captured object," J. Guid. Control, Dyn., vol. 39, no. 4, pp. 754-769, 2016.

[29] D. Mellinger and V. Kumar, "Minimum snap trajectory generation and control for quadrotors," in IEEE Int. Conf. Robotics Autom. (ICRA), 2011, pp. 2520-2525.

[30] T. Lee, M. Leok, and N. H. McClamroch, "Nonlinear robust tracking control of a quadrotor UAV on SE (3)," Asian J. Control, vol. 15, no. 2, pp. 391-408, 2013.

[31] K. Shoemake, "Animating rotation with quaternion curves," in $A C M$ SIGGRAPH computer graphics, vol. 19, no. 3. ACM, 1985, pp. 245-254.

[32] W. C. Durham, "Constrained control allocation," J. Guid. Control, Dyn., vol. 16, no. 4, pp. 717-725, 1993.

[33] S. Boyd and L. Vandenberghe, Convex optimization. Cambridge University Press, 2004.

[34] K. A. Bordignon, "Constrained control allocation for systems with redundant control effectors," Ph.D. dissertation, Virginia Tech, 1996.

[35] B. H. Wick, "Study of the subsonic forces and moments on an inclined plate of infinite span," National Advisory Committee for Aeronautics. Ames Aeronautical Lab," Technical Note 3221, 1954.

[36] L. Meier, P. Tanskanen, L. Heng, G. H. Lee, F. Fraundorfer, and M. Pollefeys, "Pixhawk: A micro aerial vehicle design for autonomous flight using onboard computer vision," Autonomous Robots, vol. 33, no. 1-2, pp. 21-39, 2012. 